\title{
Improving nursing student cultural competence: Comparing simulation to case-based learning
}

\author{
SeonYoon Chung*1, Melissa Jarvill ${ }^{2}$ \\ ${ }^{1}$ Organizational Systems and Adult Health Department, University of Maryland School of Nursing, Baltimore, MD, United States \\ ${ }^{2}$ Mennonite College of Nursing, Illinois State University, Normal, IL, United States
}

Received: February 27, 2019

DOI: $10.5430 /$ jnep.v9n $7 \mathrm{p} 128$
Online Published: April 20, 2019

\begin{abstract}
Background: Cultural competence encompasses knowledge, skills, and comfort in caring for patients from diverse cultures and is a core competency in providing patient-centered care. Simulation provides an opportunity to expose students to diverse cultures. Case-based learning has been effective in improving nursing student communication and problem-solving skills, but no research describes its use in cultural education. The purpose of this study was to compare the effect of simulation to case-based learning on nursing students' perceived cultural competence, awareness, and sensitivity.

Methods: Eighty baccalaureate nursing students were randomly assigned to a simulation experience or case-based learning exercise. The Cultural Competence Assessment Survey was used to measure perceived cultural competence, awareness, and sensitivity.

Results: Both simulation and case-based learning improved nursing student perceived cultural awareness and sensitivity. Casebased learning improved perceived cultural competence.

Conclusions: Integration of cultural learning opportunities into nursing education provides a foundation for continued development of cultural competence.
\end{abstract}

Key Words: Cultural competence, Cultural awareness, Cultural sensitivity, Simulation, Nursing students, Nursing education

\section{INTRODUCTION}

Cultural competence encompasses knowledge, skills, awareness, and comfort in caring for patients from diverse cultures. ${ }^{[1,2]}$ Nurses and nursing students work with patients and families from diverse backgrounds and thus cultural competence is specified as a core competency in providing patient-centered care. ${ }^{[3]}$ Educating nursing students about culturally competent care is necessary so they may provide quality care and improve patient outcomes for diverse populations. ${ }^{[4,5]}$ Commonly provided cultural education includes guest lectures, study abroad programs, group discussions, and written reports; yet, there is no consensus on the most effective strategies to improve the cultural competence of nurses. ${ }^{[6]}$

Simulation provides students an experiential learning opportunity ${ }^{[7]}$ and is a widely accepted innovative teaching strategy to complement clinical learning in nursing education. ${ }^{[8]}$ During debriefing sessions, facilitators provide performance feedback and explore student perspectives, which allows students to reflect on their performance and examine how they may improve future performance. ${ }^{[9]}$ Simulation and debriefing sessions provide opportunities to expose students

\footnotetext{
*Correspondence: SeonYoon Chung; Email: seonyoonchung@umaryland.edu; Address: Organizational Systems and Adult Health Department,
} University of Maryland School of Nursing, Baltimore, MD, United States. 
to diverse cultures and may improve their ability to provide culturally competent patient care. ${ }^{[10]}$ Cultural diversity can be incorporated into simulation scenarios by using culturally specific names, language, clothing, and jewelry to increase realism. ${ }^{[11]}$ Harder $^{[12]}$ reported computer-based or manikinbased simulations provide physically or socially constrained cultural context, indicating standardized patients may be useful in simulation-based learning. The use of standardized patients in cultural competence education of graduate nursing students has been beneficial in improving cultural knowledge, ${ }^{[13]}$ increasing confidence, and decreasing anxiety. ${ }^{[14]}$

Case-based learning is a learner-centered, collaborative, and active teaching strategy designed to help learners develop critical thinking skills. ${ }^{[15]}$ Nursing students are presented with realistic cases to analyze and develop plans and solutions for patient problems. ${ }^{[15,16]}$ Nursing students report the use of case-based learning contributes to their problemsolving abilities, critical thinking skills, and understanding of relationships between patient data and patient care. ${ }^{[16,17]}$ Case-based learning has also been shown to be more effective than traditional lecture in improving nursing student communication skills, problem-solving skills, and motivation to learn. ${ }^{[18]}$

Both simulation and case-based learning are used in the education of baccalaureate nursing students. In a review of literature of the role of simulation in cultural competence education, Foronda and colleagues ${ }^{[19]}$ described a lack of studies using clear sampling processes, validated instruments, and robust study designs. San ${ }^{[11]}$ also suggested studies using validated instruments and pretest-posttest designs are needed to support the use of simulation to enhance the cultural education of nurses. No research was found describing the use of case-based learning in cultural education of nursing students. Thus, the purpose of this study was to compare the effect of cultural simulation to cultural case-based learning on baccalaureate nursing students' perceived cultural competence, awareness, and sensitivity.

\section{METHOD}

\subsection{Participants and sampling procedures}

A convenience sample $(\mathrm{N}=80)$ of baccalaureate nursing students were recruited from an introductory medical-surgical nursing course at a large Midwestern public university. These nursing students were selected because they completed a cultural course in the previous semester, but had limited exposure to cultural diversity in the clinical setting. All students enrolled in the selected course were assigned to participate in either the cultural simulation experience or cultural casebased learning as part of the course requirements; however, only data from consented students were used for the purpose of this study. Of 93 students enrolled in the medical-surgical nursing course, 80 participated in the study representing $86 \%$ of the class.

The university institutional review board approved the study and authors of the Cultural Competence Assessment Sur$v^{2} y^{[20]}$ granted permission to modify and use the survey. The course faculty allowed the researchers to implement the study during the regularly scheduled simulation activities in fall 2017.

\subsection{Design}

A quasi-experimental two group pretest posttest design was used to compare the effect of a cultural simulation experience versus case-based learning on nursing student perceived cultural competence, awareness, and sensitivity. Each clinical group was randomly assigned by one of the researchers to the cultural simulation experience $(\mathrm{n}=38)$ or the cultural case-based learning exercise $(n=42)$. Prior to the cultural activities, students completed a preparatory written assignment. Each student completed the demographic survey, pretest, an assigned cultural experience, and a posttest, which all took place in the university's nursing simulation laboratory.

\subsection{Instruments}

The pretest included the Cultural Competence Assessment Survey, a 26-item Likert-type instrument composed of a single self-reported Cultural Competence Assessment (CCA) item, an 11-item cultural awareness and sensitivity (CAS) subscale, and a 14-item cultural competence behaviors (CCB) subscale to measure cultural competence in nursing students. ${ }^{[20,21]}$ Higher scores represent greater knowledge, more positive attitude, and more culturally appropriate behaviors. "No opportunity" was added as an answer choice to the CCB because students may not have previous practice experience to report. The CCB was collected only during the pretest to compare the baseline competence behaviors between groups and to describe the overall sample. The posttest included the CCA and the CAS subscale. The reliability (Cronbach alpha: $\mathrm{CCA}=.89-.92, \mathrm{CAS}=.75, \mathrm{CCB}=.91)$, construct validity, and content and face validity are well established for this instrument. ${ }^{[20-22]}$ The Cronbach alpha for this study was .75 and .66, pre and post respectively.

\subsection{Program implementation}

\subsubsection{Cultural simulation experience}

Nursing students designated to the cultural simulation experience group participated in a 20-minute simulation scenario and 20-minute debriefing. After orientation to the simulated hospital and process, the facilitator provided bedside 
report. For the purpose of this study, a previously established scenario in the nursing simulation program was modified to include cultural components and evaluated using Waxman's ${ }^{[23]}$ scenario validation checklist. The scenario featured a 60-year old female Arab Muslim standardized patient, wearing a hijab and abaya, who had hip surgery and needed a dressing change. The scenario objectives were to identify, prioritize, and implement nursing assessments and interventions for a culturally diverse patient. The students were expected to assess cultural needs of the patient, protect the patient's modesty during the dressing change, and offer a Halal compliant diet as a substitute to the general diet ordered in response to the patient's request. During the scenario, two nursing students worked together to provide care for the patient. Two additional nursing students observed the scenario via an audio-video system, took notes on the nursing simulation laboratory's standard observer form, and participated in the debriefing.

All simulation experiences were facilitated by one of the researchers, who is a nurse educator formally trained in simulation facilitation and debriefing. The NLN Jeffries Simulation Theory ${ }^{[7]}$ and the International Nursing Association of Clinical Simulation and Learning (INACSL) Standards of Best Practice for SimulationSM ${ }^{[24]}$ guided the implementation of the cultural simulation experience. Debriefings were structured based on the Debriefing for Meaningful Learning model ${ }^{[9]}$ and facilitated using the Debriefing with Good Judgment philosophy. ${ }^{[25]}$

\subsubsection{Cultural case-based learning}

Nursing students designated to the cultural case-based learning group participated in a 40-minute case-based learning exercise in groups of four. One of the researchers, who is a nurse educator formally trained in simulation facilitation and debriefing facilitated all case-based learning exercises. The case featured the same patient and situation as the cultural simulation experience. The case-based learning objectives were to identify and prioritize nursing assessments and interventions for a culturally diverse patient. Students were expected to address how to protect the patient's modesty and cultural dietary needs. Consistency between the simulation experience and the case-based learning exercise ensured that all students were exposed to the same content.

\subsection{Data analysis}

SPSS version 22.0 (IBM Corporation, Armonk, NY) was used to perform statistical analysis. Descriptive statistics were used to analyze students' characteristics. Paired $t$-test was used to determine the significance of improvement from pre to post intervention. Independent $t$-test was used to determine the difference in students' characteristics between groups and the difference in the improvement within the groups.

\section{RESULTS AND DISCUSSION}

On average, students were 21 years old, with a range from 19 to 36 years old. The majority of students were female $(87.5 \%)$ and Caucasian (83.3\%). More than half of the students had healthcare experience as a patient care technician or nursing assistant (32.5\%), volunteer (20\%), medical office assistant (3.8\%) and/or pharmacy technician (1.3\%). There were no significant differences in demographic characteristics, pretest self-reported CCA, CAS, or CCB scores between the simulation and case-based learning groups.

\subsection{Cultural competence behaviors}

The CCB average was $3.43(\mathrm{SD}=1.01$, range $=1.14-5.43)$, indicating cultural competence behaviors were reported as sometimes or often demonstrated. The CCB average positively correlated with pre $(r=.338, p=.002)$ and post CAS scores $(r=.371, p=.001)$. This finding was not surprising because individuals with greater cultural sensitivity and awareness are more likely to demonstrate culturally appropriate behaviors based on that knowledge and attitude than individuals with less cultural awareness and sensitivity.

\subsection{Perceived cultural competence}

Scores on the single item self-reported CCA, "Overall, how competent do you feel working with people who are from cultures different than your own?" improved in both groups (see Table 1). The magnitude of improvement was significantly larger in the case-based learning group compared to the simulation group, $t(77)=2.562, p=.013$. The improvement was statistically significant only in the case-based learning group. It is possible that nursing students who participated in the cultural case-based learning may have rated their cultural competence high because they were reflecting on a discussion, rather than their actual performance. In contrast, nursing students assigned to the cultural simulation group may have rated their competence low because they participated in an experiential learning opportunity and debriefing where they had an opportunity to be self-aware of actual performance and reflective of areas for improvement. This aligns with findings from studies in the healthcare field reporting that less-experienced trainees or novice practitioners tend to over-or-underestimate their competence compared to those with more experience or training who demonstrate higher self-assessment precision. ${ }^{[26,27]}$ 
Table 1. Cultural Competence Assessment (CCA) improvement from pre to post-intervention

\begin{tabular}{llllllll}
\hline \multirow{2}{*}{ Groups } & \multicolumn{2}{l}{ Pre-intervention } & & \multicolumn{2}{l}{ Post-intervention } & \multicolumn{2}{l}{ Statistics Within Groups } \\
\cline { 2 - 3 } & $\mathbf{n}$ & Mean (SD) & & $\mathbf{n}$ & Mean (SD) & $\boldsymbol{t}(\boldsymbol{p})$ \\
\hline Case-based Learning & 37 & $3.73(.902)$ & & 37 & $4.14(.536)$ & $-2.751(.009 *)$ \\
Simulation & 41 & $3.98(.821)$ & & 41 & $4.02(.612)$ & $-.350(.728)$ \\
\hline
\end{tabular}

${ }^{*} p<.05$; CCA $=5$-point Likert; SD = Standard Deviation.

\subsection{Cultural awareness and sensitivity}

CAS subscale scores improved significantly in both groups (see Table 2). The cultural simulation experience group had a greater improvement in the CAS subscale scores than the cultural case-based learning group, however, there was no significant difference between the groups, $t(78)=-1.369, p$ $=.175$. Cohen's effect size value for the simulation group $(\mathrm{d}=.52)$ indicated a medium effect and for the case-based learning group $(\mathrm{d}=.28)$ indicated a small to medium ef- fect. The trend suggests the benefit of experiential learning and debriefing in developing cultural awareness and sensitivity. The use of Debriefing for Meaningful Learning as a debriefing structure provides an opportunity for students to develop reflection and clinical reasoning skills. ${ }^{[9]}$ In this case, students in the cultural simulation group not only discussed nursing care for this specific patient during debriefing, but also how knowledge might be applied for patients with different cultural needs.

Table 2. Cultural Awareness and Sensitivity (CAS) improvement from pre to post-intervention

\begin{tabular}{|c|c|c|c|c|c|}
\hline \multirow{2}{*}{ Groups } & \multicolumn{2}{|c|}{ Pre-intervention } & \multicolumn{2}{|c|}{ Post-intervention } & \multirow{2}{*}{$\begin{array}{l}\text { Statistics Within Groups } \\
t(p)\end{array}$} \\
\hline & $\mathbf{n}$ & Mean (SD) & $\mathbf{n}$ & Mean (SD) & \\
\hline Case-based Learning & 38 & $5.94(.575)$ & 38 & 6.09 (.499) & $-2.908\left(.006^{*}\right)$ \\
\hline Simulation & 42 & $5.97(.509)$ & 42 & $6.22(.443)$ & $-4.999(<.001 *)$ \\
\hline
\end{tabular}

Note. ${ }^{*} p<.05$; CAS $=7$-point Likert; $\mathrm{SD}=$ Standard Deviation

\subsection{Program impact}

As a result of conducting this study, we became aware of the lack of cultural elements in our simulation program. Incorporating cultural elements into an already established simulation scenario was less burden than creating an entirely new scenario. We plan to incorporate cultural elements into other established simulation scenarios in our program.

\section{Conclusion}

Integration of cultural learning opportunities into nursing education is essential to provide a foundation for continued development of cultural competence. Findings from this study indicated both simulation and case-based learning improved nursing student perceived cultural awareness and sensitivity and case-based learning improved nursing student perceived cultural competence. Including cultural components in simulation scenarios provides students with an experiential context and debriefing offers students an opportunity to develop clinical reasoning and reflective skills to impact future practice. Debriefing questions that specif- ically facilitate exploration of students' cultural sensitivity, awareness, and behaviors may be helpful.

\section{ACKNOWLEDGEMENTS}

The authors would like to thank Lynn Kennell, an instructional assistant professor at Illinois State University (ISU) Mennonite Colleges of Nursing (MCN), for her thoughtful review of the scenarios used in this study and Blanca Miller, an assistant professor at ISU MCN, for her help with recruiting study participants. Appreciation also goes to Marcia Buchs, a coordinator of, and Carley Zwart, Jordyn Woods, and Chad Cuconato, assistants in the MCN Clinical Simulation Laboratory, for their help preparing and running the simulations. Thanks to all the students who participated in this study. The authors also appreciate assistance from In-Seo La, a graduate student at University of Maryland School of Nursing, with the final edits and formatting of the manuscript.

\section{Conflicts of InTEREST Disclosure}

The authors declare that there is no conflict of interest.

\section{REFERENCES}

[1] Giger J. Transcultural nursing: Assessment and intervention. St. Louis, MO: Elsevier Health Sciences; 2016.
[2] Leininger M, McFarland M. Transcultural nursing-concepts, theories, research and practice. New York, NY: McGraw Hill Companies, Inc.; 2002 
[3] Cronenwett L, Sherwood G, Barnsteiner J, et al. Quality and safety education for nurses. Nurs Outlook. 2007 May; 55(3): 122-31. https://doi.org/10.1016/j.outlook.2007.02.006

[4] American Association of Colleges of Nursing. Cultural competency in nursing education. 2016 [cited 2016 Oct 2]. Available from: http://www. aacn.nche.edu/education-resources/c ultural-competency

[5] National League for Nursing. NLN center for diversity and global initiatives. 2016 [cited 2016 Oct 2]. Available from: http://www.nln.org/centers-for-nursing-education/n ln-center-for-diversity-and-global-initiatives

[6] Long TB. Overview of teaching strategies for cultural competence in nursing students. J Cult Divers. 2012; 19(3): 102-8.

[7] Jeffries PR, Rodgers B, Adamson K. NLN Jeffries simulation theory: Brief narrative description. Nurs Educ Perspect. 2015 Sep/Oct; 36(5): 292-3. https://doi.org/10.5480/1536-5026-36.5.292

[8] Breymier TL, Rutherford-Hemming T, Horsley TL, et al. Substitution of clinical experience with simulation in prelicensure nursing programs: A national survey in the United States. Clin Simul Nurs. 2015 Nov; 11(11): 472-8. https://doi.org/10.1016/j.ecns .2015 .09 .004

[9] Dreifuerst KT. Getting started with debriefing for meaningful learning. Clin Simul Nurs. 2015 May; 11(5): 268-75. https://doi or g/10.1016/j.ecns. 2015.01.005

[10] Haas B. Incorporating cultural diversity and caring through simulation in a baccalaureate nursing program. International Journal of Human Caring. 2005; 14(2): 50-51. https : //doi .org/10. 20467 /1091-5710.14.2.50

[11] San EO. Using clinical simulation to enhance culturally competent nursing care: A review of the literature. Clin Simul Nurs. 2015 Apr; 11(4): 228-43. https://doi.org/10.1016/j.ecns . 2015.01.0 04

[12] Harder N. Determining the effects of simulation on intercultural competency in undergraduate nursing students. Nurse Educ. 2018 Jan/Feb; 43(1): 4-6. https ://doi.org/10.1097/NNE. 00000000 00000403

[13] Ndiwane A, Koul O, Theroux R. Implementing standardized patients to teach cultural competency to graduate nursing students. Clin Simul Nurs. 2014 Feb; 10(2): e87-94. https ://doi.org/10.101 6/j.ecns.2013.07.002

[14] Rutledge CM, Garzon L, Scott M, et al. Using standardized patients to teach and evaluate nurse practitioner students on cultural competency. Int J Nurs Educ Scholarsh. 2004 Sep; 1(1): Article 17. https://doi.org/10.2202/1548-923X. 1048

[15] Savery. Overview of PBL: Definitions and distinctions. Interdiscip J Probl Learn. 2006; 1(1): 9-20.
[16] Forsgren S, Christensen T, Hedemalm A. Evaluation of the case method in nursing education. Nurse Educ Pract. 2014 Mar; 14(2): 164-9. https://doi.org/10.1016/j.nepr.2013.08.003

[17] Kantar LD, Massouh A. Case-based learning: What traditional curricula fail to teach. Nurse Educ Today. 2015 Aug; 35(8): e8-14. https://doi.org/10.1016/j.nedt.2015.03.010

[18] Yoo MS, Park HR. Effects of case-based learning on communication skills, problem-solving ability, and learning motivation in nursing students. Nurs Heal Sci. 2015 Jun; 17(2): 166-72. https : //doi.org/10.1111/nhs.12151

[19] Foronda CL, Baptiste DL, Pfaff T, et al. Cultural competency and cultural humility in simulation-based education: An integrative review. Clin Simul Nurs. 2018 Feb; 15: 42-60. https://doi.org/ 10.1016/j.ecns.2017.09.006

[20] Schim SM, Doorenbos AZ, Miller J, et al. Development of a cultural competence assessment instrument. J Nurs Meas. 2003; 11(1): 29-40. https://doi.org/10.1891/jnum.11.1.29.52062

[21] Doorenbos AZ, Schim SM, Benkert R, et al. Psychometric evaluation of the cultural competence assessment instrument among healthcare providers. Nurs Res. 2005 Sep/Oct; 54(5): 324-31. https://doi.org/10.1097/00006199-200509000-00006

[22] Doorenbos AZ, Morris AM, Haozous EA, et al. ReCAP: Assessing cultural competence among oncology surgeons. J Oncol Pract. 2016 Jan; 12(1): 61-2. https ://doi.org/10.1200/JOP.2015.00693 2

[23] Waxman KT. The development of evidence-based clinical simulation scenarios: Guidelines for nurse educators. J Nurs Educ. 2010 Jan; 49(1): 29-35. https://doi.org/10.3928/01484834-2009091 6-07

[24] The International Nursing Association of Clinical Simulation and Learning (INACSL) Standards Committee. INACSL Standards of Best Practice: SimulationSM. Clin. Simul. Nursing. 2016 Dec; 12: S48-50. https://doi.org/10.1016/j.ecns.2016.10.001

[25] Rudolph JW, Simon R, Rivard P, et al. Debriefing with good judgment: Combining rigorous feedback with genuine inquiry. Anesthesiol Clin. 2007 Jun; 25(2): 361-76. https : //doi.org/10.1016/j . anclin .2007 .03 .007

[26] Quick JA, Kudav V, Doty J, et al. Surgical resident technical skill self-evaluation: increased precision with training progression. J Surg Res. 2017 Oct; 218: 144-9. https ://doi.org/10.1016/j.jss . 2017.05 .070

[27] Scaffidi MA, Grover SC, Carnahan H, et al. Impact of experience on self-assessment accuracy of clinical colonoscopy competence. Gastrointest Endosc. 2018 Mar; 87(3): 827-36. https : //doi.org/10.1016/j.gie.2017.10.040 